\title{
$-O$ HIV/AIDS, Risk Aversion and Intertemporal Choice
}

Judith Lammers'

Sweder van Wijnbergen ${ }^{2}$

1 Tilburg University;

2 University of Amsterdam. 


\section{Tinbergen Institute}

The Tinbergen Institute is the institute for economic research of the Erasmus Universiteit Rotterdam, Universiteit van Amsterdam, and Vrije Universiteit Amsterdam.

Tinbergen Institute Amsterdam

Roetersstraat 31

1018 WB Amsterdam

The Netherlands

Tel.: $\quad+31(0) 205513500$

Fax: $\quad+31(0) 205513555$

Tinbergen Institute Rotterdam

Burg. Oudlaan 50

3062 PA Rotterdam

The Netherlands

Tel.: $\quad+31(0) 104088900$

Fax: $\quad+31(0) 104089031$

Most TI discussion papers can be downloaded at http:/ /www.tinbergen.nl. 


\title{
HIV/AIDS, Risk Aversion and Intertemporal Choice
}

\author{
By
}

\author{
Judith Lammers and Sweder van Wijnbergen \\ Tilburg University University of Amsterdam
}

October 2007, revised, January 2008

\begin{abstract}
This study analyses the relation between perceived health status and intertemporal choice. We use data from experiments with real monetary rewards conducted among students in South Africa to estimate risk and time preferences. These experimental data, based on multiple price lists developed by Coller \& Williams (1999), Holt \& Laury (2002), and Harrison et al. (2002, 2005a), show that HIV+ agents and participants that perceive to have a high HIV contraction risk are less risk-averse. Although the latter group displays higher discount rates, HIV positive agents seem to have substantially lower discount rates, indicating longer time horizons in spite of their lowered life expectancy. However, we show that direct estimates of discount rates can be seriously biased estimators of the pure rate of time preference when other factors than just the pure rate of time preference are not considered simultaneously. We correct for differential mortality risk, risk aversion and differences in anticipated future marginal utility increases and price in these factors when calculating pure rates of time preference from observed discount rates. Once these factors are taken into account, HIV+ agents' time preferences conform to expectations.
\end{abstract}

Keywords: discount rate, risk aversion, perceived HIV infection risk, mortality, time preferences, marginal utility, hyperbolic discounting

This paper builds on and uses data from an earlier research project, conducted by the first author in collaboration with Morten Lau (Durham Business School) and Harrie Verbon (Tilburg University). We thank the Netherlands Organization for Scientific Research (NWO), CentER (Tilburg University), and the Durham Business School for their funding of the earlier project and NWO and CentER for funding the work reported in this paper. We also thank Harrie Verbon and Morten Lau for their collaboration in the first stage of this research and Marten van Garderen and Martine Smit for their support in the field. Furthermore we thank the staff of North West University, Center for the Study of AIDS (Pretoria University) for their comments and help in the organization of the research, in particular Prof. W.A. Naude, Prof. S.N. Mashego, Neo Mabille, Elana Olivier and Jason Wessenaar. Furthermore, we thank Marta Serra Garcia for her help in processing the data. 


\section{Introduction}

HIV/AIDS is the leading cause of death among young adults in Africa. Much of the international effort to help has focused on improving access to ARV treatment. Even in relatively developed South Africa, only $21 \%$ of those in need of ARV treatment have access to it (WHO, 2006); for the largest part of Africa access to treatment is even more restricted. But with 3 million people getting infected by HIV each year in Sub-Saharan Africa alone (WHO, 2006), prevention too has to play a crucial role if the disease is ever to be brought under control. Risky sexual behavior (promiscuity, unprotected sex) significantly increases the probability of infection. Therefore, knowledge of why people engage in such practices in spite of the potentially very serious consequences they may lead to, is crucial for the design of effective prevention programs. If it is ignorance, education should be the main focus of such programs. But if people persevere with unsafe sex simply because they are less riskaverse or value the future less than those that do not, education may well be insufficient.

Risk aversion and time preference are likely to have an impact: unsafe sex increases the risk of getting infected, so the more risk-averse one is, the more one should be willing to take precautions to reduce infection risks. Equally, unsafe sex trades off current benefits (presumably) against future costs; thus the more the future is discounted, the less the weight one attaches to avoiding those costs.

There is an extensive literature linking HIV infection to socio-demographic characteristics. For example, Pettifor et al. (2004) and Harris \& Van Aardt (2007) find that young singles and tertiary educated are overrepresented among those infected with HIV, but so are low socio-economic class persons. But literature that associates sexual behavior with quantified risk and time preferences scarcely exists. Using hypothetical questions and survey data in the US, Chesson et al. (2006) show that time preferences are significantly associated with a range of sexual behaviors and experiences, like ever having had sex, having sex before the age 16 years, and past or current pregnancies. However, they do not find significant differences in discount rates for individuals infected with HSV-2 ${ }^{1}$ (a sexual transmittable disease). Moreover, the use of hypothetical questions has become controversial as a way to elicit preference parameters. Holt \& Laury (2002) find significantly different answers between hypothetical experiments and experiments with real pay-offs.

\footnotetext{
${ }^{1}$ Herpes simplex virus type 2 .
} 
We therefore use data generated from trials using actual monetary rewards, conducted among students in South Africa, to investigate the relation between risky sexual behavior and risk and time preferences. ${ }^{2}$ In these trials, students were asked to make a series of choices between alternatives with different risk characteristics and timing of (real monetary) rewards. They were also asked to provide extensive information about their health status, economic circumstances, and sexual behavior.

Both HIV+ students and students who perceive to be highly at risk of contracting the virus, appear to be less risk-averse. However, with respect to time preference, in an initial analysis a paradoxical result emerges: Although there is weak evidence of a positive correlation between risky sexual behavior and discount rates, people who are actually infected seem to have much lower discount rates than those who are not ${ }^{3}$. More thorough analysis shows that this result is due to the implicit assumption made by most researchers in this field, that only the pure rate of time preference features in the pricing of future benefits. We show that once other factors than just the pure rate of time preference are incorporated in the pricing of future benefits, this seeming anomaly disappears and the results conform to prior expectations. Differential expectations about mortality rates, risk attitude and future disposable income levels turn out to be major explanatory factors of differences in valuing future events and explain most of the puzzling results on rates of time preference earlier researchers have obtained.

Similarly, we also show that conclusions on hyperbolic discounting become biased when failing to incorporate the other factors mentioned in the analysis of intertemporal choice. On uncorrected data, the hypothesis of hyperbolic discounting is accepted, but once differential expectations on mortality rates, risk preferences and future levels of disposable income are incorporated, the hypothesis is rejected.

The paper is organized as follows. The next section provides the theoretical framework for eliciting risk and time preferences. Section 3 presents the experimental method and the results assuming that only the pure rate of time preference influences the

\footnotetext{
2 These experiments were conducted in two rounds as part of the $\mathrm{PhD}$ research of the first author under the guidance of professors Harrie Verbon and Lex Meijdam of Tilburg University. Morten Lau and Harrie Verbon collaborated in the design, implementation and analysis of the first round interviews (Lammers et al. (2006)). The first round was conducted among a general recruited student sample, the second round was conducted among HIV+ students recruited from HIV support groups in order to obtain a larger group of HIV+ subjects in the sample..

${ }^{3}$ In their analysis of the first round results Lammers et al. (2006) also find this paradoxical result.
} 
pricing of future benefits, which leads to the seeming paradox on HIV status and discount rates. Section 4 introduces mortality, risk attitude and differential expectations about future consumption levels into the analysis and tests for hyperbolic discounting. Section 5 concludes.

\section{Eliciting risk and time preferences: a standard approach}

We assume that expected utility theory (EUT) holds for choices over risky alternatives and that subjects have a constant relative risk aversion (CRRA) utility function defined over the prizes they make choices over:

$$
U\left(M_{t}\right)=\frac{E M_{t}^{1-\gamma}}{1-\gamma}
$$

where $U\left(M_{t}\right)$ is the utility of a monetary outcome $M_{t}$ at time $t$ and where $\gamma$ is the CRRA coefficient. $E$ is the expectations operator. For $\gamma=1$, this function is defined as $U\left(M_{t}\right)=E \ln \left(M_{t}\right)$, for $\gamma=0$ the agent is risk-neutral, for $\gamma>0$ the agent is risk-averse, and for $\gamma<0$ the agent is risk-seeking. Furthermore, assume that discounting is exponential. Consider two certain monetary outcomes $(M)$ at time $t$ and at time $t+k$. An agent is indifferent between these two outcomes if the following equation holds:

$$
\begin{aligned}
& U\left(M_{t}\right)=D(k) U\left(M_{t+k}\right) \\
& \text { where } \quad D(k)=\frac{1}{(1+\rho)^{k}}
\end{aligned}
$$

$U\left(M_{t}\right)$ is again the utility of monetary outcome $M_{t}$ at time $t$ as specified in Equation (1). $k$ is the horizon for late delivery of monetary outcome $M_{t+k} . D(k)$ is the discount function, which can be interpreted as the relative weight an agent attaches to utility of $M_{t+k}$ at time $t+k$ compared to utility of $M_{t}$ at time $t$, and $\rho$ the pure rate of time preference. ${ }^{4}$ If agents are risk-neutral (i.e. no curvature of $U, \gamma=0$ ), Equation (2) can be written as follows:

\footnotetext{
${ }_{4}^{4}$ The pure rate of time preference measures the preference for immediate utility over delayed utility.
} 


$$
M_{t}=\frac{1}{(1+\rho)^{k}} M_{t+k}
$$

If we know agents value two monetary outcomes $M_{t}$ and $M_{t+k}$ equally in spite of their timing difference, we can derive the implicit value of $\rho$ for which (3) holds as equality. We will initially assume risk neutrality to derive this implicit value, in line with earlier literature (Harrison et al. (2002) and Coller \& Williams (1999)), but will relax this assumption later on.

\section{Experimental data}

We use data from experiments conducted among students of the North West University and the University of Pretoria in South Africa. Lammers (2008) provides a detailed description of the experimental procedures used. These build on the risk aversion tasks of Holt \& Laury (2002) and the discount rate tasks of Coller \& Williams (1999), and closely follow the experimental procedures of a Danish field experiment by Harrison et al. (2002, 2005a).

\section{Stimuli}

Each student was asked to complete one risk aversion (RA) task and six discount rate (DR) tasks (see Appendix, Table A1.1 and A1.2 for a detailed description). Each task involved a series of binary choices, in the RA task 10 and in the DR tasks 20 per task. In the RA task, subjects were asked to choose between two risky lotteries, where the probability of winning the higher price increased along the table. The point at which subjects switch from the less risky to the more risky option can be used to deduce the subject's risk preference parameter. In the six DR tasks, subjects were asked to choose between two certain outcomes: a present and a future payment. The various DR tasks differed in the timing of the future payment: the delay increased from 1 to $3,5,11,17$, and finally 23 months. If not specified differently, we study the elicited discount rates from the longest time horizon, i.e. the DR task in which the delayed payment option was 23 or 24 months. Also in the DR tasks, the point at which the subject switches puts a bound on his discount rate. For each individual, we estimate the unconditional discount rate by taking the average discount rate when a subject switches from choosing the current to the future payment option. If subjects 
switch more than once between the two options, the discount rate is assumed to be equal to the midpoint of the interval over which the subject is indifferent.

\section{Treatments}

There is empirical evidence that agents are more impatient about immediate delays than they are about future delays of the same length (Coller \& Williams, 1999). To test that hypothesis, the timing of the present option was varied between treatments, keeping the time period between the two alternatives constant. 38\% of the subjects was asked to choose between receiving an amount today or at various moments in the future, labeled No FrontEnd-Delay (ND). The alternative option was offered in 1, 3, 5, 11 or 23 months. The other subjects were asked to choose between two future options, labeled Front-End-Delay (D): the first payment option was in 1 month, and the alternative in 2, 4, 6, 12, 18 or 24 months, such that the length between the treatments remained the same in both sets of experiments. This allows us to test for (quasi)-Hyperbolic Discounting (HD).

\section{Motivating participants}

Real incentives were used to motivate participants. In addition to the fixed participation fee of 30 Rand (1 Rand equals about 0.14 USD in the year of the experiments), all subjects had a 10\% chance of being selected for actual payment according to the choice they made in each of the two tasks. The additional expected payoff in the valuation tasks was 65 Rand. This performance-based random lottery real incentive system is nowadays used by most researchers as incentive structure for individual choice experiments (Holt \& Laury, 2002). ${ }^{5}$ Subjects selected for additional payment in the DR task, received a postdated check issued by Tilburg University, which could be cashed at any Standard Bank in South Africa any time after the specified date.

\section{Questionnaires/sample characteristics}

Subjects were asked to fill out three different questionnaires: a socio-demographic, a financial and a health questionnaire. Among other health related issues, subjects were asked

\footnotetext{
5 The main advantage of this system is that it avoids income effects such as Thaler \& Johnson's (1990) house money effect. It has been shown empirically that players do not interpret choice tasks rewarded with the random lottery incentive system as one grand overall lottery (Cubitt et al. 1998, Starmer \& Sugden 1991).
} 
to report on their HIV status, and on how they perceive their chances of getting HIV infected during their lifetime. In the latter, they were asked to choose between: no risk at all (1), small (2), moderate (3), and high (4). In this paper, we analyze differences in risk and time preferences among 5 groups: the first four are based on the subject's self-reported perceived HIV contamination risk, group 5 consists of subjects who have actually contracted HIV.

\section{Participants}

Our total sample includes 213 subjects, $53.5 \%$ males, and $46.5 \%$ females. $85.0 \%$ of the respondents are non-white and $15.0 \%$ are white South African students. The age-range among white participants is smaller compared to non-white participants, ranging between 19-24 and 18-36 respectively, with a mean age of 21.1 years for white participants and 22.9 years for non-white participants. The overall reported HIV prevalence rate among our sample is $9.8 \%{ }^{6}$. Another $4.7 \%$ of the subjects indicated to prefer not to report their HIV status or to answer the question about whether or not they had ever been tested for the virus. This prevalence rate is comparable with the average prevalence rate (9.9\%) among the youth in the North West province (Pettifor et al., 2004), but the observed prevalence rate is high if we consider their finding that among the youth that are HIV positive only $10 \%$ is also aware of their status. The reported perceived contamination risk varied among the sample. For instance, $10.4 \%$ of the respondents perceived their HIV contamination risk as high, and $23.3 \%$ indicated that they would face no risk at all to contract the virus. The largest proportion (44.8\%) answered to have a small and $11.6 \%$ reported to have a moderate risk of contracting HIV during their lifetime.

\section{Sample selection criteria}

From the 213 subjects, 1 subject did not reveal his perception of HIV contamination risk, 4 subjects did not completely fill out the discount rate task, 14 subjects did not reveal their expected age of death, and 36 participants did not answer consistently ${ }^{7}$ in the RA task. Removing these subjects from the sample leaves us with 163 subjects. The discount rates

\footnotetext{
${ }^{6}$ Note that if we would not consider the second round of experiments, this percentage would be $3.0 \%$.

7 With "not consistently" we mean that subjects choose the smaller prize when both options were sure (i.e. option A in decision row 10, in Table A1.1).
} 
elicited from the 163 subjects we consider, do not significantly differ from those elicited from the subjects removed from the sample $\left(p-v^{2}\right.$ alue $\left.{ }^{8}=0.68\right)$.

\section{Results I: attitude towards risk}

Using the switching-point in the RA tasks to elicit the risk aversion parameter, we found a CRRA coefficient $(\gamma)$ of 0.16 on average, which is low compared to the student population of the subjects in Holt \& Laury (2002) ( $\gamma=0.66$, USA), or Harrison et al. (2005b) $\left(\gamma=0.54^{9}\right)$. However, Tanner et al. (2005) found on average even lower values in their crosscultural study for Niger (ranging between -0.15 and 0.14). It is intriguing that studies estimating risk preferences from asset prices using CAPM find much higher values than are obtained in experimental studies (see for example Cochrane (2001)).

Table 1 shows that the CRRA coefficient is statistically different for the 5 groups based on HIV status and perceived HIV contamination risk ( $\mathrm{p}$-value $=0.02)$. The results also suggest a negative relation between perceived HIV contraction risk and risk aversion. A simple regression gives a slope coefficient of -0.13 with $\mathrm{p}$-value 0.03 . In fact for the two highest groups, $\gamma$ is actually negative on average, indicating risk loving or a convex utility function.

Table 1: CRRA coefficients $(\gamma)$

\begin{tabular}{lcrr}
\hline \hline Perceived HIV contraction risk & \multicolumn{1}{l}{ N } & $\gamma$ & Std. Dev. \\
\hline Group 1: no risk at all & 38 & 0.24 & 0.56 \\
Group 2: small & 73 & 0.26 & 0.68 \\
Group 3: moderate & 19 & 0.18 & 0.67 \\
Group 4: high & 17 & -0.17 & 0.24 \\
Group 5: HIV+ & 16 & -0.20 & 0.70 \\
\hline All & 163 & 0.16 & 0.64 \\
\hline \hline
\end{tabular}

\section{Results II: rate of time preference}

The mean (annualized) discount rate implied by subjects' choices for the longest time horizon is $39.2 \%$, although there is substantial variation around that mean (see Table 2 ). In this section, we interpret this value as an estimate of the pure rate of time preference. This

\footnotetext{
${ }^{8}$ Based on Mann-Whitney test (see for example Siegel \& Castellan (1988).

$9 \gamma=0.54$ is the average CRRA coefficient elicited in Ethiopia, Uganda, India.
} 
estimate is much higher than, for example, real market borrowing rates in South Africa, which were around $2.3 \%{ }^{10}$ at the time when the experiment was conducted.

Analyzing the results for different groups separately produces a surprising result. Although Table 1 showed a clear negative relation between risk aversion and the risk grouping of contracting HIV, the relation found between discount rate and the risk group of those who actually contracted HIV does not seem to conform with expectations at all (see Table 2).

Table 2: Discount rate, $\rho$ (in \%)

\begin{tabular}{lccc}
\hline \hline Perceived HIV contraction risk & $\mathrm{N}$ & $\rho$ & Std. Dev. \\
\hline Group 1: no risk at all & 38 & 40.7 & 22.4 \\
Group 2: small & 73 & 41.4 & 20.2 \\
Group 3: moderate & 19 & 40.0 & 22.8 \\
Group 4: high & 17 & 44.3 & 20.7 \\
Group 5: HIV+ & 16 & 19.6 & 21.8 \\
\hline All & 163 & 39.2 & 22.0 \\
\hline \hline
\end{tabular}

Thus, experimental data seem to reject that HIV+ agents have higher discount rates. In fact, the opposite relation holds: HIV+ agents have significantly lower discount rates $(\Delta=-21.73$, $\mathrm{p}$-value $=0.0006$, where $\Delta$ equals the difference between $\mathrm{HIV}+$ agents and all other agents). Table 2 and Figure 1, however, do show an admittedly weak positive link between risk exposure and discount rates among the groups that have not yet actually contracted the virus: discount rates of agents that perceive to be highly at risk of getting infected are higher than those of agents that perceive not to be highly at risk. Drawing a regression line through the data points of the first four groups only, weakly confirms the expected positive relation between the rate of time preference and perceived HIV contraction risk. A regression yields a slope coefficient of +0.93 , but with a high $\mathrm{p}$-value (0.37). Including the fifth data point of HIV+ subjects leads to a negative slope, although also not significantly $(-3.93$, p-value $=0.26) .{ }^{11}$ The discount rate of the HIV + group seems to be an outlier in the expected positive relation between discount rate and perceived contamination risk.

\footnotetext{
${ }^{10}$ Based on inflation and prime rates 2005, source: EcoWin.

${ }^{11}$ Removing however any other of the 4 data points leads to a distinct negative slope.
} 
Figure 1: Rate of time preference ${ }^{12}$

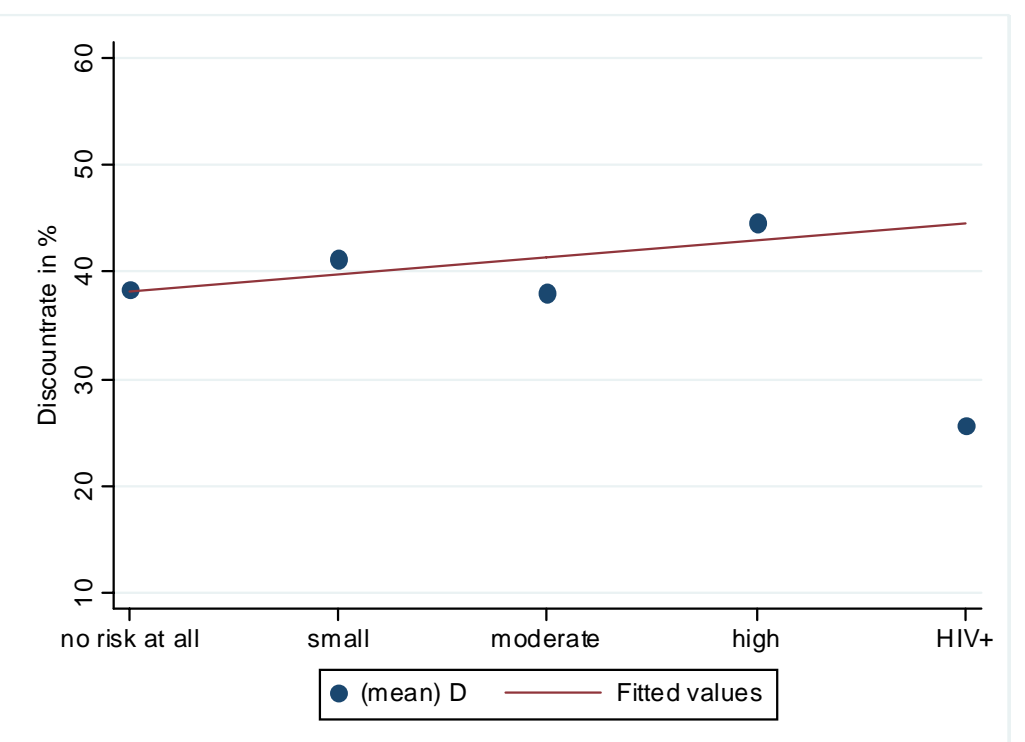

We are thus left with a puzzle. The relation between coefficients of risk aversion and risk exposure do conform to expectations, but the results on the rate of time preference do not. While there seems to be a weak positive link between increasing risk exposure and discount rates, including HIV+ agents reverses that link. HIV+ subjects actually have significantly lower discount rates. This is remarkable because considering sexual behavior, we do find results in our data that point in a different direction. For instance, sexually active agents display higher discount rates $(\rho(\operatorname{sex})=40.07(\mathrm{n}=133)$ vs. $\rho$ (no sex $)=35.47(\mathrm{n}=30)$, pvalue $=0.15)$, be it that the difference is not statistically significant. ${ }^{13}$ Furthermore, agents that are sexually active and do not take preventive measures, i.e. they do not regularly use condoms, have significantly higher discount rates $(\rho$ (no condom) $=51.71 \quad(n=14)$ vs. $\rho($ condom $)=38.07(\mathrm{n}=106), \mathrm{p}$-value $\left.=0.06^{14}\right)$. Since these behavioral variables are strongly and positively correlated with the risk of contracting HIV, why do we not find this relation for $\mathrm{HIV}+$ agents in our data?

\footnotetext{
${ }^{12}$ Regression line through the first four data points, i.e. excluding the HIV+ group.

13 Excluding the HIV+ group the difference is statistically significant $\rho$ (sex)=42.87 (n=117) vs. $\rho$ (no sex $)=35.47(\mathrm{n}=30)$, $\mathrm{p}$-value $=0.04$.

${ }^{14}$ Excluding the HIV+ group this difference is not significant: $\rho$ (no condom) $=51.71$ (n=14) vs. $\rho$ $($ condom $)=41.20(\mathrm{n}=91), \mathrm{p}$-value $=0.15$.
} 


\section{Explaining the Time Preference Paradox}

The results on discount rates and risk classes obtained so far seem paradoxical. However, the assumption that the pure rate of time preference is the only factor entering the pricing of future benefits is limiting and very likely to bias the results. We first consider differential mortality risk as an additional factor entering the relevant discount rate and then various ways of incorporating anticipated changes in marginal utility of consumption over time. We will find that the results change significantly once these additional factors are taken into account.

\subsection{Correcting for mortality risk}

An individual cannot derive utility from consumption in a certain period unless he has survived the preceding periods. Therefore, uncertainty of survival leads households to discount the future more heavily (Yaari, 1965). HIV obviously decreases actual life expectancy of HIV+ agents. Especially in developing countries where the availability of medicines is insufficient, being HIV infected means premature death. ${ }^{15}$ Variation in mortality risk may therefore explain differences in the implied individual discount rates among different risk groups.

Suppose agents have an annual survival probability $p$. Then the probability of surviving $k$ periods ahead equals $S_{t+k}=p^{k-t}$. Equation (4) then shows the mortality risk inclusive discount rate $\left(\rho^{u c}\right)$ an agent will use in period $t$ to price her well-being in period $t+k$, where $\rho$ equals the pure rate of time preference, conditioned on staying alive.

$$
\left(\frac{1}{1+\rho^{u c}}\right)^{k}=\frac{S_{t+k}}{S_{t}(1+\rho)^{k}}
$$

Substituting the survival function in (4) and solving for the pure rate of time preference, gives:

$$
\rho=\left(1+\rho^{u c}\right) p-1
$$

\footnotetext{
${ }^{15}$ In South Africa $21 \%$ of people with advanced HIV infection receives antiretroviral therapy (WHO, World Health Statistics 2006). Median time from seroconversion (clinical latency) to AIDS in east Africa is estimated to be 9.4 years. The median survival time after the progression to AIDS is 9.2 months (Morgan et al., 2002).
} 
Although we do not know $p$ for the specific sample of people considered, our health questionnaire did include the question "how old do you think you will become?" from which we can elicit the expected time until death. Under the simplifying assumption of a constant annual survival probability, there is a simple relation between survival probability $p$ and the expected time until death, $E\left(T_{D}\right)$, so that $p$ can be calculated (see Annex 2.1):

$$
p=\frac{E\left(T_{D}\right)-1}{E\left(T_{D}\right)}
$$

Since $p$ is increasing in $E\left(T_{D}\right)$, the unconditional discount rate, as presented in Table 2 , is biased upwards if interpreted as an estimate of the pure rate of time preference, as was done in Section 3. Not correcting for mortality risk will therefore create a bias in the results when comparing groups with different perceptions of their own HIV contamination risk, since they are likely to have different expected survival times.

Table 3 shows that the expected time until death indeed varies across the five groups and is decreasing in perceived HIV contraction risk (corr $=-0.32$, p-value $=0.0000)$. Average expected time until death at the time of the experiment is 49.1. The difference is highly significant across the five groups based on the Kruskal-Wallis test ( $\mathrm{p}$-value=0.0001). HIV+ subjects estimate the time until death on average 30.3 years from now, which is substantially lower (by 22.2 years) than the expected life time of the group who thinks to have no risk at all to contract the virus during their life time. This shows awareness of the lifetime reducing consequences of HIV infection.

Table 3: Expected time until death, $E_{0}\left(T_{D}\right)$

\begin{tabular}{lcccc}
\hline \hline Perceived HIV contraction risk & $\mathrm{N}$ & $E_{0}\left(T_{D}\right)$ & Std. Dev. & $\alpha$ \\
\hline Group 1: no risk at all & 38 & 52.5 & 18.5 & 0 \\
Group 2: small & 73 & 52.3 & 16.1 & 0.01 \\
Group 3: moderate & 19 & 48.6 & 18.6 & 0.18 \\
Group 4: high & 17 & 46.2 & 15.9 & 0.28 \\
Group 5: HIV+ & 16 & 30.3 & 11.6 & 1 \\
\hline All & 163 & 49.1 & 17.7 & \\
\hline \hline
\end{tabular}


Assume that group 1 has no risk of contracting the virus, so infection risk $\alpha_{1}=0$. Group 5 is already infected, so $\alpha_{5}=1$. We can express expected lifetime of the other 3 groups by Equation (7) below and elicit the corresponding infection probabilities, $\alpha_{i}$, of these groups. Both the infection probability of the moderate group $\left(\alpha_{3}=0.18\right)$ and the weighted average $(\bar{\alpha}=0.15)$ are close to the adult prevalence rate of South Africa, which was $18.8 \%$ (UNAIDS, 2006) at the time the experiments were conducted.

$$
E_{0}\left(T_{D}\right)_{G i}=\left(1-\alpha_{i}\right) E_{0}\left(T_{D}\right)_{G 1}+\alpha_{i} E_{0}\left(T_{D}\right)_{G 5}
$$

However, although our subjects seem to consider HIV infection risk in their expectations of lifetime, it appears that for 3 out of the 5 groups subjects not having medical insurance, on average estimated their lifetime higher than those with medical insurance. Apparently, these groups underestimate the consequences of infection on expected lifetime without appropriate treatment.

Based on the individual expected remaining lifetime, a survival probability $p$ is calculated; we then apply the correction for mortality when estimating time preferences using Equation (5). Table 4 shows that correcting for mortality using Equation (5) decreases the estimate of the rate of time preference on average by 1.2 percentage points. The correction differs across the five groups; even though $p$ is the lowest for the HIV+ group, the correction for this group is the lowest due to their relatively low "raw" discount rate. Correcting for mortality, however, reduces the discrepancy between the estimated discount rates of those subjects that perceive to be highly at risk and HIV + subjects $(0.28$ percentage points).

We conclude that in countries with large differences in mortality risk, as in high HIV prevalence countries like South Africa where 18.8\% of the adult population is infected (UNAIDS, 2006), not correcting for mortality in eliciting individual discount rates biases estimates for time preferences and distorts the comparison between groups in society based on individual characteristics. On the horizons considered, however, the corrections are not large compared to the underlying estimates, and the counterintuitive result for HIV + agents is still there. 
Table 4: Rate of time preference before $\left(\rho^{u c}\right)$ and after $(\rho)$ correction discount rates for differential mortality rates.

\begin{tabular}{lccc}
\hline \hline Perceived HIV contraction risk & $\mathrm{N}$ & Mean & Std. Dev. \\
\hline Group 1: no risk at all & 38 & 40.7 & 22.4 \\
$\rho^{u c}$ & 38 & 39.2 & 22.3 \\
$\rho$ & & 1.56 & \\
$\rho^{u c}-\rho$ & & & \\
Group 2: small & 73 & 41.4 & 20.2 \\
$\rho^{u c}$ & 73 & 40.4 & 19.8 \\
$\rho$ & & 1.0 & \\
$\rho^{u c}-\rho$ & & & \\
Group 3: moderate & & & \\
$\rho^{u c}$ & 19 & 40.0 & 22.8 \\
$\rho$ & 19 & 39.0 & 22.1 \\
$\rho^{u c}-\rho$ & & 1.0 & \\
Group 4: high & & & \\
$\rho^{u c}$ & 17 & 44.3 & 20.7 \\
$\rho$ & 17 & 43.1 & 20.1 \\
$\rho^{u c}-\rho$ & & 1.2 & \\
Group 5: HIV + & & & \\
$\rho^{u c}$ & 16 & 19.6 & 21.8 \\
$\rho$ & 16 & 18.7 & 20.6 \\
$\rho^{u c}-\rho$ & & 0.9 & \\
\hline All & & & \\
$\rho^{u c}$ & 163 & 39.2 & 22.0 \\
$\rho^{u c}-\rho$ & & 38.1 & 21.6 \\
\hline \hline
\end{tabular}

\subsection{Relaxing the assumption of risk neutrality}

Table 1, including estimates for risk preferences, may point part of the way to a solution to the puzzle: so far we have assumed risk neutrality in the calculation of the rate of time preference, but this table shows that risk tolerance differs across groups and is significantly higher among high risk groups. Andersen et al. (2005) show that joint elicitation of the CCRA coefficient and discount rate substantially lower discount rates compared to eliciting the two separately. Under the expected utility framework used here, the same parameter measuring risk aversion also measures (the inverse of) intertemporal substitution elasticity. Assuming risk neutrality (linear utility) therefore could have biased the elicited discount rate for these agents. 
Combining Equations (2) and (4) leads to the following estimate for the rate of time preference corrected for mortality and curvature (see Appendix 2.2 for the derivation):

$$
\rho=\left[\frac{S_{t+k}}{S_{t}}\left(\frac{M_{t+k}}{M_{t}}\right)^{1-\gamma}\right]^{1 / k}-1
$$

If we assume that the intertemporal substitution parameter (inverse of the intertemporal rate of substitution) is equal to the risk parameter obtained from the static risk experiment ${ }^{16}$, we can calculate $\rho$. Note however that, while $\gamma<0$ is possible in the context of risk, $\gamma<0$ is not an admissible value in the context of intertemporal choice. For one thing, it would imply that the first order conditions determining intertemporal choice correspond to a welfare minimum instead of a welfare maximum. We therefore restrict this part of our analysis to individuals with $\gamma \geq 0$, which means removing 73 risk-seeking subjects from our sample. The discount rate for the group with $\gamma<0$ is not significantly different from the value obtained for the group with $\gamma \geq 0$ ( $\mathrm{p}$-value $=0.18)$, whether the correction for mortality risk is applied or not.

Table 5 below shows that the correction for the "lower-risk groups", i.e. the first three groups, is substantial: 20.2 percentage points, which is in line with Andersen et al. (2005), who finds an average reduction of 15.1 percentage points among the Danish population. Furthermore, correcting for utility curvature widens the gap between the group of agents that perceive to be highly at risk of contracting HIV and the other agents, i.e. their discount rate turns out to be relatively high. Finally, it brings the implied rate of time preference of HIV + agents closer to the lower-risk group. Table 5 shows that correcting for risk attitude decreases the estimate of the rate of time preference for HIV+ agents with 3.4 percentage points. For the other groups this difference is much higher, and ranges between $5.5-24.5$ percentage points.

\footnotetext{
16 As is always the case within the expected utility framework.
} 
Table 5: Discount rates corrected for mortality only $\left(\rho^{M}\right)$ and for mortality and risk attitude $(\rho)$. Sample with $\gamma \geq 0$.

\begin{tabular}{|c|c|c|c|}
\hline Perceived HIV contraction risk & $\mathrm{N}$ & Mean & Std. Dev. \\
\hline \multicolumn{4}{|l|}{ Group 1: no risk at all } \\
\hline$\gamma$ & 23 & 0.58 & 0.43 \\
\hline$\rho^{M}$ & 23 & 37.2 & 24.1 \\
\hline$\rho$ & 23 & 19.4 & 17.3 \\
\hline$\rho^{M}-\rho$ & & 17.8 & \\
\hline \multicolumn{4}{|l|}{ Group 2: Small } \\
\hline$\gamma$ & 46 & 0.64 & 0.50 \\
\hline$\rho^{M}$ & 46 & 42.5 & 20.7 \\
\hline$\rho$ & 46 & 21.8 & 22.0 \\
\hline$\rho^{M}-\rho$ & & 20.73 & \\
\hline \multicolumn{4}{|l|}{ Group 3: Moderate } \\
\hline$\gamma$ & 8 & 0.79 & 0.59 \\
\hline$\rho^{M}$ & 8 & 49.2 & 27.1 \\
\hline$\rho$ & 8 & 24.6 & 29.9 \\
\hline$\rho^{M}-\rho$ & & 24.5 & \\
\hline \multicolumn{4}{|l|}{ Group 4: High } \\
\hline$\gamma$ & 4 & 0.16 & 0.11 \\
\hline$\rho^{M}$ & 4 & 56.5 & 21.7 \\
\hline$\rho$ & 4 & 51.0 & 24.6 \\
\hline$\rho^{M}-\rho$ & & 5.5 & \\
\hline \multicolumn{4}{|l|}{ Group 5: HIV+ } \\
\hline$\gamma$ & 9 & 0.25 & 0.26 \\
\hline$\rho^{M}$ & 9 & 18.0 & 17.3 \\
\hline$\rho$ & 9 & 14.6 & 14.7 \\
\hline$\rho^{M}-\rho$ & & 3.4 & \\
\hline \multicolumn{4}{|l|}{ All } \\
\hline$\gamma$ & 90 & 0.58 & 0.48 \\
\hline$\rho^{M}$ & 90 & 39.9 & 23.1 \\
\hline$\rho$ & 90 & 22.0 & 21.8 \\
\hline$\rho^{M}-\rho$ & & 17.9 & \\
\hline
\end{tabular}

Figure 2 shows the average discount rates before (DM) and after correction for risk attitude (DRA) for the 5 different groups. Both sets of points have also been corrected for differential mortality expectations. The figure illustrates the importance of incorporating risk preferences in estimating time preferences. Figure 2 does show a trend upwards. The average discount rates for HIV+ agents, however, is still lower compared to uninfected subjects, 
although not significantly anymore $(\mathrm{p}$-value $=0.32)$. This correction again reduced the discrepancy between the HIV+ group and the group that perceives to be highly at risk (2.1 percentage points), but this difference remains significant $(p$-value $=0.02)$.

In summary, our data show that after correcting for mortality and curvature of the intertemporal utility function, agents that perceive to be more at risk of contracting HIV, do display significantly higher rates of time preference as well as lower coefficients of risk aversion. Nevertheless, HIV+ agents still display lower discount rates than those who have not (yet?) contracted the disease. However, this difference is not statistically different anymore.

Figure 2: Discount rates corrected for mortality (DM) and risk aversion (DRA) Sample with $\gamma \geq 0 .{ }^{17}$

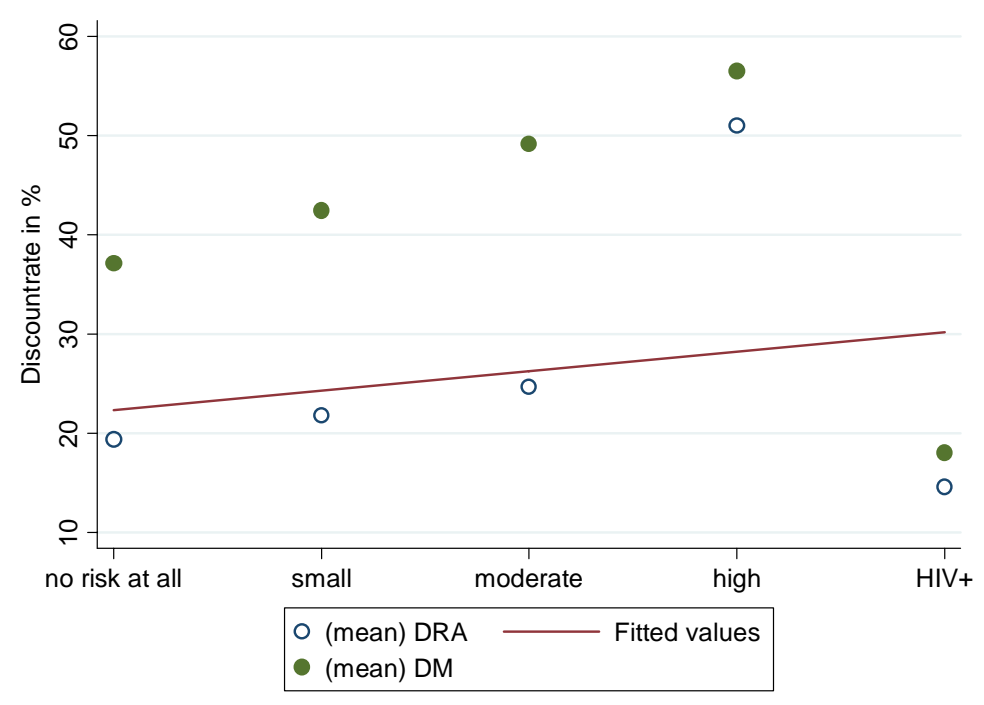

\subsection{Discount rates and future income decline}

Another possible explanation for the relatively low discount rate for HIV+ subjects could be that their expected future budget constraint is expected to be relatively tight compared to other subjects. In the analysis so far, we assumed that the monetary benefits $M$ accrued on top of a basically unchanging consumption level. But due to their (known) illness, expenditures will rise for the HIV+ group, and income will most likely fall. In that case,

${ }^{17}$ Fitted values through data points of the implied discount rate corrected for mortality and risk attitude (DRA). 
significant differences in marginal utility may occur between the two points of time over which the experiment was conducted ${ }^{18}$. If anticipated differences in future marginal utility are not fully eliminated by higher savings, the imputed discount rate will be lower than the true degree of time preference. ${ }^{19}$

Assume that utility of consumption is specified by $U(C)=\frac{C^{1-\gamma}}{1-\gamma}, \quad U^{\prime}(C)>0, U^{\prime \prime}(C)<0$. Using future marginal utility over current marginal utility to make future goods comparable to current goods, an estimate for the implied discount rate $\rho$ is obtained as specified in the following equation:

$$
\begin{gathered}
\rho=\left[\frac{S_{t+k}}{S_{t}} \frac{M_{t+k}}{M_{t}} q_{t+k}^{-\gamma}\right]^{1 / k}-1 \\
\text { where } q_{t+k}^{-\gamma}=\frac{U^{\prime}(C(t+k))}{U^{\prime}(C(t))}=\left(\frac{C(t+k)}{C(t)}\right)^{-\gamma}
\end{gathered}
$$

where $q_{t+k}$ equals the relative future consumption level $\mathrm{k}$ periods ahead in terms of the consumption level today (see Appendix 2.3 for the analytical derivation of (9)). As long as $\gamma>0$, the implied discount rate $\rho$ is decreasing in both $q_{t+k}$ and $\gamma$, i.e. ignoring that $q_{t+k}<1$ leads to a downward bias in the estimate of $\rho$.

Of course, we cannot observe $q_{t+k}$. But, we do have experimental results for students with and without medical insurance. If anticipated future medical costs are the reason for anticipating lower future consumption, it is reasonable to set $q_{t+k}=1$ for those with medical insurance since they will have their medical bills covered. The uninsured HIV+ group (NI) realistically enough estimated their own time till death 5.5 years lower than the insured HIV $+\operatorname{group}(\mathrm{I})\left(E_{0}^{I}\left(T_{D}\right)=37.5\right.$ vs. $E_{0}^{N I}\left(T_{D}\right)=31.0, \mathrm{p}$-value=0.38). Surprisingly, in our sample uninsured HIV+ subjects are on average slightly more risk-averse, although not significantly $\left(\gamma^{I}=0.14\right.$ vs. $\gamma^{N I}=0.29, \mathrm{p}$-value $\left.=0.65\right)$. Not infected uninsured subjects, however, do display significantly higher risk aversion $\left(\gamma^{I}=0.73\right.$ vs. $\gamma^{N I}=0.53$, p-value $\left.=0.04\right)$. In our total sample,

\footnotetext{
18 Olson \& Baily (1981) already stress that differences in marginal utility over time should be excluded from the definition of pure time preference.

${ }^{19}$ Botelho e.a. in the concluding section of an analysis linking preference parameters to demographic characteristics also suggest that anticipated future consumption risk (they discuss food security in Timor Leste) may influence measured preference parameters.
} 
$32.1 \%$ of our subjects were having medical insurance. Among non-whites, the percentage was significantly less, $22.2 \%$ vs. $87.5 \%$.

Our data indeed show that HIV+ subjects with medical insurance (30.4\% of the sample) have substantially higher discount rates than uninsured HIV+ subjects: ${ }^{20}$

$$
\rho^{I}=29.82 \text { vs. } \rho^{N I}=10.24 \text {, p-value }=0.14
$$

This difference enables us to estimate the anticipated decline in consumption $q$. If we assume uninsured have the same pure rate of time preference as insured participants, one can derive an estimate of the anticipated decline in consumption, $q$, from the difference in observed discount rates (see Appendix 2.3):

$$
\hat{q}_{N I}=\left(\frac{1+\rho_{N I}}{1+\overline{\rho_{I}}}\right)^{\frac{1}{\gamma_{N I}}}
$$

where $\overline{\rho_{I}}$ is the average of the for mortality and risk attitude corrected discount rate of insured HIV+ subjects as defined in Equation (8) and $\rho_{N I}$ is the for mortality corrected discount rate as defined in Equation (5).

This procedure yields an estimate of $\hat{q}_{N I}=0.66$, which, interestingly enough, is very close to the findings of Steinberg et al. (2002). They empirically show that in South Africa, HIV households spend a significant part of households' expenditures on medical treatment, on average $34 \%$. This corresponds to a $q$-value of $66 \%$. Using the same dataset, van de Kuilen \& Lammers (2007) show that HIV+ students save more than respondents who are not infected, and those without insurance save more than those with insurance. Apparently they do not save enough, however, to fully arbitrage expected marginal utility differences away.

Incorporating this anticipated decline in future consumption for uninsured HIV+ participants increases the estimated rate of time preference of HIV+ subjects by 14.6 percentage points compared to the discount rate with corrections for mortality only. The rate of time preference for both the HIV+ group and the high-risk group are now higher than those obtained for the lower risk groups.

\footnotetext{
20 These discount rates are already corrected for mortality and risk attitude as described in the previous section.
} 
Figure 3: Discount rate corrected for mortality, risk attitude, and relative future consumption level (DMU). Sample with $\gamma \geq 0$.

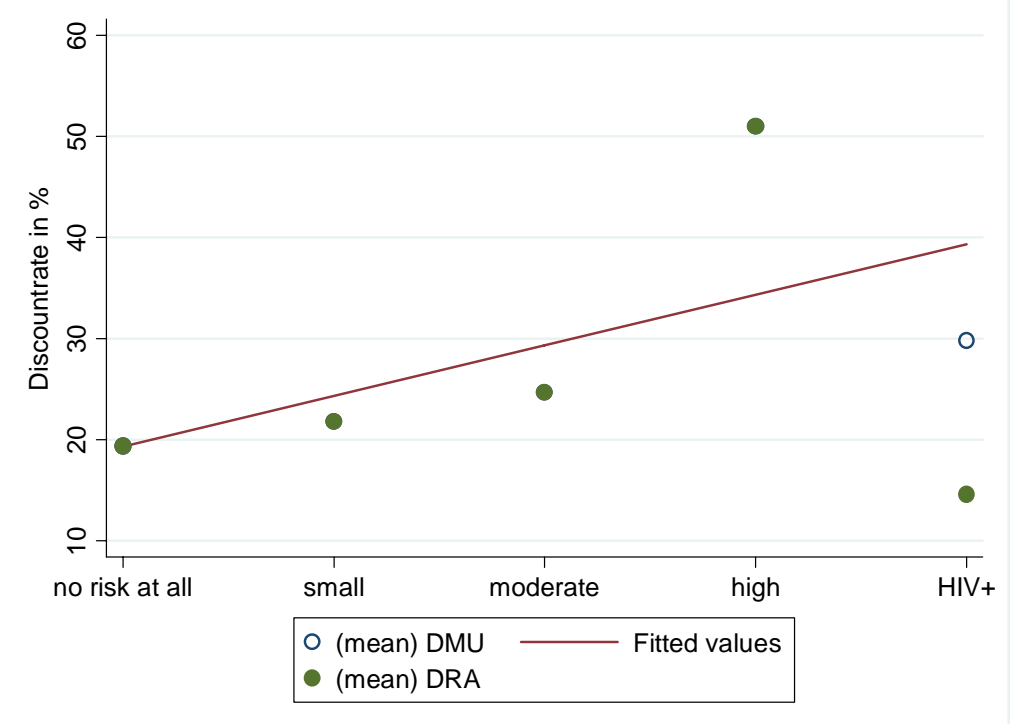

The discount rate for the high-risk group is in fact strikingly higher than the rates we found for all other groups, even the one for the HIV+ group. This may be explained by the fact that the HIV+ group probably does not exclusively consists of people who belonged to the high-risk group before their infection. Less risky behavior lowers the chance of getting infected, but not to zero, since there are various other ways of transmission which may not be related to risky behavior, and anyhow less risky behavior reduces risk but does not always reduce it to zero.

And after this correction, the implied discount rate of the HIV+ group now fits in a pattern of rates of time preference rising with perceived risk exposure. Figure 3 shows the average discount rates before (DRA) and after the correction for marginal utility (DMU). The slope becomes steeper $(+5.01)$, although it remains insignificant ( $p$-value $=0.26)$.

To summarize, uninsured HIV+ subjects seem to consider the fall in future marginal utility due to expected illness costs when prizing future benefits. Correcting their discount rate for differences in mortality risk, risk attitude, and the difference in anticipated future marginal utility substantially increases the estimated rate of time preference of HIV positive subjects. These corrections reverse the earlier result that found them having a significantly lower rate of time preference than the other groups. After corrections, that is not the case anymore. 


\subsection{Correcting for quasi-hyperbolic discounting}

There is evidence that people, when choosing between options having both shortrun and long-run consequences, tend to overvalue short-run consequences and downplay long term costs, for example in unprotected sex (O’Donoughue \& Rabin (2000)). Our experiments also allow for testing this hypothesis of Quasi-Hyperbolic Discounting (QHD), because subjects were randomly assigned the choice between two alternative testing scenarios, differing only because of a time shift of one month in all the choices offered. ${ }^{21}$

There is another reason to check for (QHD). The subjects within each group were randomly assigned to the delay treatment, so in principle the difference in task should not have a significant effect when comparing average discount rates between groups, as we have done so far. However, the randomized assignment procedure had as unfortunate outcome that in both the HIV+ group and the high-risk group, only one respondent was assigned the treatment with immediate gratification. Analysis of group averages, and in particular the results for these two groups, could therefore still be biased by a QHD effect, if there is any, since the delayed option experiments were overrepresented in these groups.

An analysis of the means of the "raw" data (implicit rates of time preferences not corrected for differential mortality risk or curvature of the utility function) seemingly supports the QHD hypothesis. Participants seem to be more impatient when choosing between immediate and postponed gratification than when choosing between two delayed gratifications, holding the time span constant. For the longest time horizon experiments (23 months) the difference in the mean discount rate was 12.5 percentage points on average and is statistically significant: the Mann-Whitney test indicates that the two sample are not drawn from the same distribution $(\mathrm{p}-\mathrm{value}=0.02)$.

However, replicating the analysis after correcting for both mortality and curvature of the utility function changes the results. The difference between estimated rates of time preference of the group with and without delayed gratification then turns out to become both much smaller and insignificant $(\Delta=3.40, \mathrm{p}$-value=0.71). Apparently, ignoring mortality risk and curvature of utility in the calculation of time preference biases the test for QHD. We also tested for QHD within each risk group, using the specification of quasi-hyperbolic discount functions suggested by O’Donoghue \& Rabin (1999). This did not lead to different conclusions: QHD is rejected for all subgroups.

${ }^{21}$ We again restrict the analysis to subjects with a nonnegative risk parameter. 
The conclusion seems clear. On direct estimates of discount rates, the test for QuasiHyperbolic Discounting is accepted, but that result evaporates once corrections for betweengroup-differences in mortality, attitudes towards risk and anticipated future consumption decline are incorporated in the estimation of the discount rate $\rho$. We conclude that it is not necessary to further correct our estimates of time preference for the treatment effect in analyzing the relation between time preference and perceived HIV contraction risk.

\section{Conclusions}

This paper studies whether risky behaviour leading to increased HIV contraction risk can be explained by risk and time preferences. Since unsafe sex increases the risk of getting infected, intuitively the more risk-averse one is, the more one should be willing to take precautions to reduce infection risks. Equally, unsafe sex trades off current benefits against future costs; thus the more one discounts the future, the less the weight one attaches to avoiding those costs.

Using raw data from economic experiments with real monetary rewards, we do find that risk aversion is significantly and negatively related to perceptions of HIV contraction risk. However, no such relation is found for raw estimates of time preferences. While risky sexual behavior is correlated with higher discount rates, HIV+ respondents seemingly but paradoxically displayed significantly more patient behavior in choosing between present and future payment options than all other groups considered.

However, the assumption that the pure rate of time preference is the only factor entering the pricing of future benefits, though commonly made in the experimental literature, is limiting and appears to bias the results. Therefore, this paper considered other factors entering the relevant discount rate when estimating time preferences. Incorporating differences in perceived mortality risk, risk attitude and anticipated changes in marginal utility of consumption over time, reverses the initial finding that HIV+ respondents would have significantly lower discount rates than the other groups. The applied corrections decreased the discrepancy between the discount rates of the high-risk group and the HIV+ group by 17.9 percentage points and is no longer statistically different. We even find the expected distinct positive relation between discounting and perceived exposure to contracting HIV. Applying the corrections thus seems to solve the paradox. 
Our experimental data also allowed the estimation of the decline in future consumption levels that HIV+ infected respondents expect. This estimate is similar to what Steinberg et al. (2002) report on the share of medical expenses in total consumption of HIV affected households. Although Van de Kuilen \& Lammers (2007) find higher saving rates among HIV+ respondents than in the rest of the sample, HIV+ respondents apparently do not save enough to significantly offset anticipated future declines in consumption opportunities.

This paper thus shows that superficial analysis of time preferences, i.e. without correcting for other factors relevant in the pricing of future benefits, can be very misleading when comparing different risk groups in society. In particular, we show the importance of differences in expected mortality, risk attitude and expected increases in marginal utility of consumption over time. The relevance of this work should be clear given the current trend of conducting experiments in the field.

We conclude that risk and time preference not only have an impact on risky sexual behavior, but that they are also related to perceptions of HIV contraction risk. In addition, estimations for the average infection probabilities based on perceptions of remaining lifetime are close to the actual HIV prevalence rate in South Africa at the time the experiments were conducted. Our experimental data thus suggest that our respondents do not continue to practice unsafe sex because of ignorance, but because they are less risk-averse and value the future less than those that do not. Accordingly, prevention focused on education alone is likely to be insufficient.

Even accepting that risky behaviour reflects risk preferences and a low rate of time preference rather than ignorance about risk factors, their behavior may still be privately suboptimal if they underestimate the total expected illness costs. Since for three out of the five risk groups the average expected lifetime is higher for the medically uninsured subsamples, this indeed suggests that although individuals are aware of HIV contraction risk itself, they seem to underestimate the consequences of HIV infection, such as the costs and need for medical treatment. Therefore, providing information about the actual illness costs might be another useful action to deter individuals from risky sexual behavior.

Finally, since there are high social costs attached to HIV/AIDS, intervention seems justified even if individuals are fully informed and act privately optimal. Considering the high rate of time preference we found, offering monetary incentives might be necessary to tilt the 
intertemporal tradeoff implicit in choosing to practice unsafe sex towards the safe sex option. An example of such an incentive would be free distribution of condoms, as part of a campaign to prevent the further spread of HIV. Clearly, this measure alone is unlikely to be enough, since the students in the sample already had easy access to free condoms at the campus.

\section{References}

Andersen, Steffen; Harrison, Glenn W.; Lau, Morten I.; \& Rutström, E. Elisabet (2005). "Eliciting Risk and Time Preferences." Working paper 05-24, Department of Economics, College of Business Administration, University of Central Florida, USA, forthcoming, Econometrica.

Botelho, A., G. Harrison, L. Costa Pinto, E. Rutström and P. Veiga (2006), "Discounting in Developing Countries: Experimental Evidence from Timor Leste”, Working Paper 05-31, Department of Economics, College of Business Administration, University of Central Florida, USA

Chesson, Harrell W.; Jami S. Leichliter, Gregory D. Zimet, Susan L. Rosenthal, David I. Bernstein, \& Kenneth H. Fife (2006). "Discount rates and risky sexual behaviors among teenagers and young adults," Journal of Risk and Uncertainty, Springer, vol. 32(3), May, p. 217-230.

Cochrane, John H. (2001) Asset Pricing. -- Princeton University Press.

Coller, Maribeth, \& Melonie B. Williams, (1999). "Eliciting Individual Discount Rates," Experimental Economics, 2, p. 107-127.

Cubitt, Robin. P., Chris Starmer \& Robert Sugden (1998). "On the Validity of the Random Lottery Incentive System,” Experimental Economics, 1, p. 115-131.

Frederick, Shane, George Loewenstein \& Ted O'Donoghue (2002). “Time Discounting and Time Preference: A Critical Review." Journal of Economic Literature, American Economic Association, vol. XL, June, p. 351-401.

Harris, M. \& C.J. Van Aardt (2007). Fusing HIV/Aids risk segmentation and demographic modelling: a novel way of looking at the business impact of HIV/Aids in South Africa. Paper given at SABCOHA breakfast on January 30, Johannesburg.

Harrison, Glenn W., Morten I. Lau, Elisabet Rutström, \& Melonie B. Sullivan (2005a). 
"Eliciting Risk and Time Preferences Using Field Experiments: Some Methodological Issues," in J. Carpenter, G. W. Harrison and J. A. List (eds.), Field Experiments in Economics (Greenwich, CT: JAI Press, Research in Experimental Economics, Volume 10).

Harrison, Glenn W., Steven J. Humprey, \& Arjen Verschoor (2005b). "Choice Under Uncertainty: Evidence from Ethiopia, India and Uganda," Working Paper 05-29, Department of Economics, College of Business Administration, University of Central Florida, USA.

Harrison, Glenn W.; Lau, Morten I., \& Williams, Melonie B. (2002). "Estimating Individual Discount Rates in Denmark: A Field Experiment," American Economic Review, vol.92(5), December, p. 1606-1617.

Holt, Charles A., \& Susan K. Laury (2002). "Risk Aversion and Incentive Effects," American Economic Review, 92(5), December, p. 1644-1655.

Kuilen. Gijs Van de. \& Judith Lammers (2007). "HIV contraction risk and the anticipatory saving motive: an empirical study in South Africa," CentER Discussion Paper No. 200751 , July.

Lammers, J, Lau M.I, \& Verbon, H.A.A. (2006). Perceived HIV contamination risk, risk aversion and time preferences (a laboratory experiment in South Africa, conference paper, FUR XII 2006 at LUISS, June 2006. Available at http://center.uvt.nl/phd_stud/lammers/188.pdf

Lammers, Judith (2008). "HIV/AIDS, risk and intertemporal choice" CentER Dissertation series NR 209, ISBN 978905668910 1, Tilburg University, Forthcoming. Appendix available at http://center.uvt.nl/phd_stud/lammers/Appendixsept26.pdf

Maital, S., Maital, S. (1977). "Time preference, delay and gratification and the intergenerational transmission of economic inequality: a behavioral theory in income distribution,” in: Ashenfeltar, O., Oates, W. (Eds.), Essays in Labor Market Analysis in Memory of Yochanan Peter Comay, Wiley Publishing, New York, p. 179-200.

O’ Donoghue, Ted \& Matthew Rabin (1999). "Doing it now or later," American Economic Review, vol. 89(1), p. 103-124.

O’ Donoghue, Ted \& Matthew Rabin (2000), "Risky Behavior Among Youths: Some Issues from Behavioral Economics,”. Economics Department, University of California, Berkeley, Working Paper E00-285. 
Olson, Mancur \& Bailey, Martin J. (1981). "Positive Time Preference," Journal of Political Economy, University of Chicago Press, vol. 89(1), pages 1-25, February.

Pettifor, Audrey E., Helen V. Rees, Annie Steffenson, Lindiwe Hlongwa-Madikizela, Catherine MacPhail, Kerry Vermaak, \& Immo Kleinschmidt (2004). HIV and sexual behaviour among young South African: a national survey of 15-24 year olds. Reproductive Health Research Unit, University of the Witwatersrand, Johannesburg.

Siegel, S. \& Castellan, N.J. Jr. (1988). Nonparametric statistics for the behavioral sciences.

McGraw-Hill, international edition 1988.

Starmer, Chris \& Robert Sugden (1991). "Does the Random-Lottery Incentive System Elicit True Preferences? An Experimental Investigation," American Economic Review, 81, p. $971-978$.

Steinberg M., S. Johnson, G. Schierhout \& D. Ndegwa (2002). “Hitting Home: How Households Cope with the Impact of the HIV/ AIDS Epidemic." A Survey of Households Affected by HIV/AIDS in South Africa, The Henry J. Kaiser Family Foundation, Washington.

Tanner, Mariah, Jayson Lusk, \& Wallace Tyner (2005). “A Multidimensional Investigation of Economic Behavior in Four Countries," Working paper, August.

Thaler, R. H. \& E. J. Johnson (1990). “Gambling with the House Money and Trying to Break Even: The Effects of Prior Outcomes on Risky Choice," Management Science 36, p. 643-660.

Yaari, M.E. (1965). "Uncertain lifetime, life insurance, and the theory of the consumer," Review of Economic Studies, vol. 32, p. 137-150.

World Health Organization (2006). World Health Statistics 2006. 


\section{Appendix 1: Tables from the experiment}

Table A1.1 The ten paired lottery choice decisions

\begin{tabular}{|c|c|c|c|c|c|c|}
\hline Decision & Option A & Option B & Option A & Option B & $\begin{array}{c}\text { Expected } \\
\text { payoff }\end{array}$ & $\begin{array}{l}\text { Open CRRA interval if } \\
\text { subject switches to } \\
\text { option B }\end{array}$ \\
\hline 1 & $\begin{array}{l}\text { R } 50.00 \text { if ball is } 1-10 \\
\text { R } 40.00 \text { if ball is } 11-100\end{array}$ & $\begin{array}{l}\mathrm{R} 96.25 \text { if ball is } 1-10 \\
\mathrm{R} \quad 2.50 \text { if ball is } 11-100\end{array}$ & R 41 & R 11,88 & 29,13 & $-\infty,-1.71$ \\
\hline 2 & $\begin{array}{l}\text { R } 50.00 \text { if ball is } 1-20 \\
\text { R } 40.00 \text { if ball is } 21-100\end{array}$ & $\begin{array}{l}\mathrm{R} 96.25 \text { if ball is } 1-20 \\
\mathrm{R} \quad 2.50 \text { if ball is } 21-100\end{array}$ & R 42 & R 21,25 & 20,75 & $-1.71,-0.95$ \\
\hline 3 & $\begin{array}{l}\text { R } 50.00 \text { if ball is } 1-30 \\
\text { R } 40.00 \text { if ball is } 31-100\end{array}$ & $\begin{array}{l}\mathrm{R} 96.25 \text { if ball is } 1-30 \\
\mathrm{R} \quad 2.50 \text { if ball is } 31-100\end{array}$ & R 43 & R 30,63 & 12,38 & $-0.95,-0.49$ \\
\hline 4 & $\begin{array}{l}\text { R } 50.00 \text { if ball is } 1-40 \\
\text { R } 40.00 \text { if ball is } 41-100\end{array}$ & $\begin{array}{l}\mathrm{R} 96.25 \text { if ball is } 1-40 \\
\mathrm{R} \quad 2.50 \text { if ball is } 41-100\end{array}$ & R 44 & $\mathrm{R} 40$ & 4 & $-0.49,-0.15$ \\
\hline 5 & $\begin{array}{l}\text { R } 50.00 \text { if ball is } 1-50 \\
\text { R } 40.00 \text { if ball is } 51-100\end{array}$ & $\begin{array}{l}\mathrm{R} 96.25 \text { if ball is } 1-50 \\
\mathrm{R} \quad 2.50 \text { if ball is } 51-100\end{array}$ & R 45 & R 49,38 & $-4,38$ & $-0.15,0.14$ \\
\hline 6 & $\begin{array}{l}\text { R } 50.00 \text { if ball is } 1-60 \\
\text { R } 40.00 \text { if ball is } 61-100\end{array}$ & $\begin{array}{l}\mathrm{R} 96.25 \text { if ball is } 1-60 \\
\mathrm{R} \quad 2.50 \text { if ball is } 61-100\end{array}$ & R 46 & R 58,75 & $-12,75$ & $0.14,0.41$ \\
\hline 7 & $\begin{array}{l}\text { R } 50.00 \text { if ball is } 1-70 \\
\text { R } 40.00 \text { if ball is } 71-100\end{array}$ & $\begin{array}{l}\mathrm{R} 96.25 \text { if ball is } 1-70 \\
\mathrm{R} \quad 2.50 \text { if ball is } 71-100\end{array}$ & R 47 & R 68,13 & $-21,13$ & $0.41,0.68$ \\
\hline 8 & $\begin{array}{l}\text { R } 50.00 \text { if ball is } 1-80 \\
\text { R } 40.00 \text { if ball is } 81-100\end{array}$ & $\begin{array}{l}\mathrm{R} 96.25 \text { if ball is } 1-80 \\
\mathrm{R} \quad 2.50 \text { if ball is } 81-100\end{array}$ & R 48 & R 77,5 & $-29,5$ & $0.68,0.97$ \\
\hline 9 & $\begin{array}{l}\text { R } 50.00 \text { if ball is } 1-90 \\
\text { R } 40.00 \text { if ball is } 91-100\end{array}$ & $\begin{array}{l}\mathrm{R} 96.25 \text { if ball is } 1-90 \\
\mathrm{R} \quad 2.50 \text { if ball is } 91-100\end{array}$ & R 49 & R 86,88 & $-37,88$ & $0.97,1.37$ \\
\hline 10 & R 50.00 if ball is $1-100$ & R 96.25 if ball is $1-100$ & R 50 & R 96,25 & $-46,25$ & $1.37, \infty$ \\
\hline
\end{tabular}

The last three columns were not presented to the subjects. 
Table A1.2 Discount rate task, Problem 6 (FED) ${ }^{22}$

\begin{tabular}{|c|c|c|c|c|c|}
\hline Decision & $\begin{array}{c}\text { Option } \mathbf{A} \\
\text { To be paid in } 1 \text { month }\end{array}$ & $\mid \begin{array}{c}\text { Option B } \\
\text { To be paid in } 24 \text { months }\end{array}$ & $\begin{array}{c}\text { Annual } \\
\text { Interest rate }\end{array}$ & \multicolumn{2}{|c|}{$\begin{array}{l}\text { Your choice } \\
\text { (Circle A or B) }\end{array}$} \\
\hline 1 & R172 & R182.60 & $3 \%$ & A & $\mathrm{B}$ \\
\hline 2 & R172 & R193.76 & $6 \%$ & A & $\mathrm{B}$ \\
\hline 3 & R172 & R205.51 & $9 \%$ & A & $\mathrm{B}$ \\
\hline 4 & R172 & R217.88 & $12 \%$ & A & $\mathrm{B}$ \\
\hline 5 & R172 & R230.90 & $15 \%$ & A & B \\
\hline 6 & R172 & R244.60 & $18 \%$ & A & $\mathrm{B}$ \\
\hline 7 & R172 & R259.00 & $21 \%$ & A & B \\
\hline 8 & R172 & R274.14 & $24 \%$ & A & B \\
\hline 9 & R172 & R290.05 & $27 \%$ & A & B \\
\hline 10 & R172 & R306.76 & $30 \%$ & A & $\mathrm{B}$ \\
\hline 11 & R172 & R324.30 & $33 \%$ & A & $\mathrm{B}$ \\
\hline 12 & R172 & R342.72 & $36 \%$ & $\mathrm{~A}$ & $\mathrm{~B}$ \\
\hline 13 & R172 & R362.05 & $39 \%$ & A & B \\
\hline 14 & R172 & R382.32 & $42 \%$ & A & $\mathrm{B}$ \\
\hline 15 & R172 & R403.58 & $45 \%$ & A & B \\
\hline 16 & R172 & R425.87 & $48 \%$ & A & B \\
\hline 17 & R172 & R449.22 & $51 \%$ & A & B \\
\hline 18 & R172 & R473.69 & $54 \%$ & A & B \\
\hline 19 & R172 & R499.32 & $57 \%$ & $\mathrm{~A}$ & B \\
\hline 20 & R172 & R526.15 & $60 \%$ & A & B \\
\hline
\end{tabular}

${ }^{22}$ The nFED task entails a choice between option A: one month and option B: 23 months. In Problem 1, payment option B entails a gratification in 2 instead of 24 months. 


\section{Appendix 2: Analytical derivations}

\section{A2.1 Corrections for mortality}

Suppose agents have an annual survival probability $S_{t+k}=p^{k-t}$ of surviving period $t+k$, where $p$ is the probability of surviving to the next period. Equation (11) then shows the relative weight an agent attaches in period $t$ to her well-being in period $t+k$ corrected for mortality from which we can elicit agents' unconditional discount rate $\rho^{u c}$, i.e. not conditioned on the survival rate.

$$
\left(\frac{1}{1+\rho^{u c}}\right)^{k}=\frac{S_{t+k}}{S_{t}(1+\rho)^{k}}
$$

where $\rho$ the discount rate conditional on his survival function $S_{t+k}$. Assuming constant survival probability, the probability of dying in year $k$ is $f(k)=p^{k}(1-p)$ such that from the expected time of death measured at time $0, E_{i}\left(T_{D}\right)=$ Lex $_{i}-A g e_{i}$, the probability of survival to the next period, $p$ can be solved using the general formula of Equation (12) for a converging infinite arithmo-geometric series, i.e.:

$$
\begin{aligned}
& \sum_{k=0}^{\infty}(a+k r) q^{k}=\frac{a}{1-q}+\frac{r q}{(1-q)^{2}} \\
E_{k}\left(T_{D}\right)= & \sum_{k=0}^{\infty} f(k) k=\sum_{k=0}^{\infty} p^{k-1}(1-p) k=\frac{1-p}{p} \sum_{k=0}^{\infty} p^{k} k \\
= & \frac{1-p}{p} \frac{p}{(1-p)^{2}}=\frac{1}{1-p} \\
\Rightarrow & p=\frac{E_{k}\left(T_{D}\right)-1}{E_{k}\left(T_{D}\right)}
\end{aligned}
$$

Substituting (13) in (11) and solving for the conditional discount rate, provides the discount rate conditional on the discrete survival function, specified in Equation (14).

$$
\rho=\left(1+\rho^{u c}\right) p-1
$$




\section{A2.2 Corrections for risk attitude}

Consider two certain monetary outcome $M_{t}$ and $M_{t+k}$ at time $t$ and $t+k$. An individual is indifferent between these two monetary outcomes if Equation (15) holds.

$$
\begin{aligned}
& U\left(M_{t}\right)=D(k) U\left(M_{t+k}\right) \\
& \text { where } U\left(M_{t}\right)=\frac{M_{t}^{1-\gamma}}{1-\gamma} \text { and } D(k)=\frac{1}{\left(1+\rho^{u c}\right)^{k}}
\end{aligned}
$$

In this specification, we drop our initially assumption that individuals are risk neutral in intertemporal choices such that $\frac{\partial U\left(M_{t}\right)}{\partial \gamma} \geq 0$ if $\gamma \geq 0$. Combining Equation (15), and (11) results in the Equation (16):

$$
\frac{S_{t} M_{t}^{1-\gamma}}{1-\gamma}=\frac{1}{(1+\rho)^{k}} \frac{S_{t+k} M_{t+k}^{1-\gamma}}{1-\gamma}
$$

Solving for $\rho$ provides us with the for mortality and risk attitude corrected discount rate:

$$
\rho=\left[\frac{S_{t+k}}{S_{t}}\left(\frac{M_{t+k}}{M_{t}}\right)^{1-\gamma}\right]^{1 / k}-1
$$

\section{A2.3 Corrections for differences in anticipated future consumption levels (for HIV+ group only)}

Assume again that individuals have a nonnegative risk parameter $\gamma \geq 0$ and that their utility is specified by $U(C)=\frac{C^{1-\gamma}}{1-\gamma}, \quad$ where $\quad U^{\prime}(C)=C^{-\gamma}>0, U^{\prime \prime}(C)=-\gamma C^{-\gamma-1}<0$ if $\gamma>0 . \quad$ If $\quad$ we furthermore assume that, for people who are actually infected by HIV, the impact on marginal utility of anticipated consumption decline is substantially larger than the impact of differences in monetary rewards, we can ignore the latter and use a standard expression for the consumption discount factor (CDF) to compare current and future award benefits: 


$$
C D F=\frac{U^{\prime}\left(C_{t+k}\right) S_{t+k}}{U^{\prime}\left(C_{t}\right) S_{t}(1+\rho)^{k}}
$$

Using (18) in the equation indicating when agents are indifferent between an award at $t$ and an award at $t+k$, yields:

$$
\begin{gathered}
S_{t} M_{t}=\frac{1}{(1+\rho)^{k}} S_{t+k} M_{t+k} q_{t+k}^{-\gamma} \\
\text { where } q_{t+k}^{-\gamma}=\frac{U^{\prime}(C(t+k))}{U^{\prime}(C(t))}=\left(\frac{C(t+k)}{C(t)}\right)^{-\gamma}
\end{gathered}
$$

in which $q_{t+k}$ is the relative future consumption level over k periods. Solving for $\rho$ provides the rate of time preference corrected for mortality risks and marginal utility differences over time.

$$
\rho=\left[\frac{S_{t+k}}{S_{t}} \frac{M_{t+k}}{M_{t}} q_{t+k}^{-\gamma}\right]^{1 / k}-1
$$

Assuming a nonnegative intertemporal substitution parameter $\gamma, \rho$ is decreasing in both $q_{t+k}$ and $\gamma$ :

$$
\frac{\partial \rho}{\partial q}=\frac{\partial \rho}{\partial \gamma}=-\frac{\gamma}{k} \frac{S_{t+k}}{S_{t}} \frac{M_{t+k}}{M_{t}} q_{t+k}^{-\gamma-1}<0
$$

Assuming that the relative price of future consumption is different for HIV + agents, but equal for all agents in each group, we can estimate $\rho$ if we know the value of $q_{t+k}$. If we furthermore assume that differences in the relative future consumption level between insured (I) and not insured (NI) HIV+ individuals are only caused by expenditures for medical consumption and that moreover insured and uninsured HIV + agents have the same pure rate of time preference, $\hat{q}_{N I}$ in Equation (22) yields an estimate for the relative future consumption level of uninsured HIV+ individuals with respect to insured HIV+ individuals: 


$$
\begin{gathered}
\left.\begin{array}{l}
\rho_{N I}^{a c t}=\left(1+\rho_{N I}^{o b s}\right) q_{N I}^{-\gamma_{N I}}-1 \\
\rho_{N I}^{a c t}=\rho_{I}^{a c t}=\rho_{I}^{o b s}
\end{array}\right\} \rho_{I}^{o b s}=\left(1+\rho_{N I}^{o b s}\right) q_{N I}^{-\gamma_{N I}}-1 \Rightarrow \\
\hat{q}_{N I}=\left(\frac{1+\rho_{N I}^{o b s}}{1+\overline{\rho_{I}^{o b s}}}\right)^{\frac{1}{\gamma_{N I}}}
\end{gathered}
$$

Where $\overline{\rho_{I}^{o b s}}$ is the average of the for mortality and risk attitude corrected discount rate of insured HIV+ subjects as defined in Equation (17) and $\rho_{N I}^{o b s}$ is the for mortality corrected discount rate as defined in Equation (14). 